\title{
The application architecture research of Internet of things based on Web FENG Liang ${ }^{1, a}$ \\ ${ }^{1}$ School of Management, Northwestern Polytechnical University, Xi'an City 710072,China \\ ${ }^{a}$ FengLiang1029@yeah.net
}

Keywords: Internet of things, Web, Web services environment

\begin{abstract}
With the development of embedded devices and in-depth research and extensive application of networking technology, smart objects and types, increasing the number of networking devices continue to be popular in the people's daily lives. Development of digital and network technologies enables the controller node, sensor nodes, radio frequency identification, and household appliances such as television, air-conditioning equipment can use the Internet protocol, wireless communication protocol to achieve information transmission and exchange. Things technology triggered a new wave of the information industry, and unlimited commercial value. Things study based on real significance of architecture and key technologies of Web applications is very important.
\end{abstract}

\section{Introduction}

With the popularity of Internet technology and embedded devices deployed a large number of research and development, make the amount and type of smart objects are in constant growth, equipment and systems in use in people's everyday life is becoming more and more popular. The evolution of network technology, also makes the sensor nodes, the controller node, RFID, and some electrical appliances (TV, alarm clock, acoustics, air conditioning, etc.) through wireless communication protocol and the Internet network interconnection and information exchange. The digital and networked intelligent devices are widely used in smart home, intelligent logistics, intelligent transportation and intelligent agriculture and other fields, has a huge commercial value. The Internet of things is, as it were, following computer, Internet and mobile communications technology triggered a wave of information industry of core areas, the developed countries to carry out the layout in the field of Internet of things.

With the Internet of Things to promote research and development and deployment of technology and a lot of embedded devices, the number and type of smart objects in the growing networking equipment and systems used in people's daily lives are becoming increasingly popular. Situation of Things application development but due Things equipment, high coupling and poor scalability issues, divisive, the threshold is high, long development cycle [1]. According to the background and problems, we propose a Web services environment (WoTSE). Its core idea is a variety of equipment resources seamless access to the Internet of Things, in the form of Web pages open, so the integration of heterogeneous networking equipment, reduce application development difficulty and networking technology, while ensuring that the system of distributed Features loose coupling and large-scale deployment [2].

\section{The technology architecture of Internet of things}

Internet of things have full perception, reliable transmission and can only process characteristics. Due to the integration of many things now appear in the latest technology, so I do not like the Internet, by the International Organization for Standardization clear hierarchy model. But the Internet also has its own hierarchy, the Internet is recognized technical architecture is divided into three layers, top to bottom are things perception layer, the network layer of the Internet of things application layer [3-4], as shown in Figure 1. 


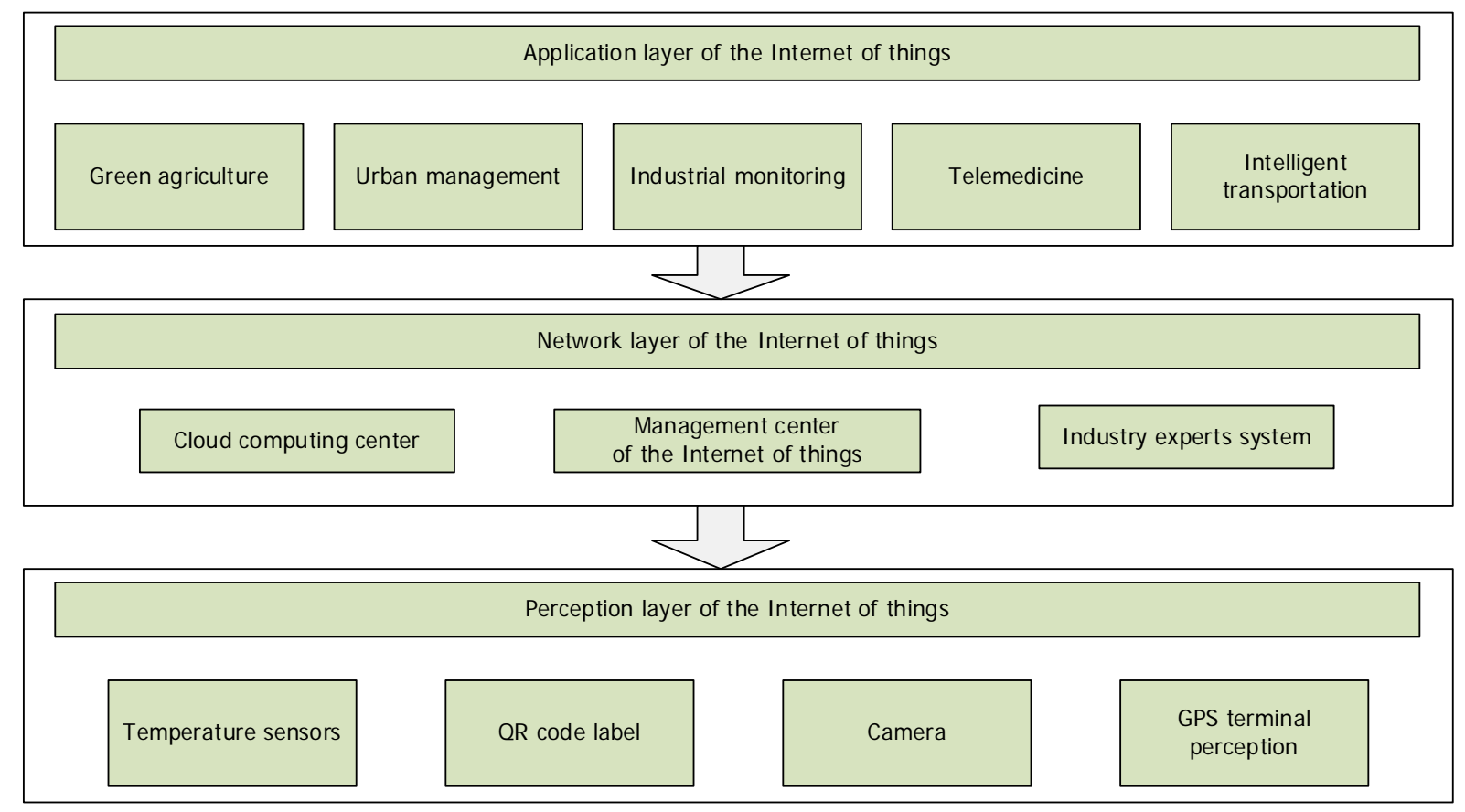

Figure 1.Technology architecture of Internet of things

Perception layer. Things sensing layer is the first layer of the three-tier technology. The role of information the real physical world and the virtual world of perception layer, the perception of the bottom of a wide variety of information by means of a temperature sensor, two-dimensional code label, camera, GPS terminals and other sensing equipment acquisition, and these physical information into digital information is transmitted to the platform.

Network layer. Internet network layer is located on the second floor. Network layer after layer function is to establish a network-aware layer and the bridge, the question of Things application layer is responsible for sales of Things perception layer to collect a variety of information, and the information accurately and efficiently delivered to the application layer of things. The network layer is based on the foundation of modern mobile communication technology and Internet technology, through a variety of wireless data transmission equipment.

Application layer. Things application layer on the third floor. The role of the application layer is to create things, wide interface, and intelligent networking applications. Integrated system functionality of the application layer at the bottom, through software development, to provide users with a friendly interface, and can achieve carrier networking applications. Things oriented application layer industries, including green agriculture, industrial monitoring, urban management, telemedicine and so on.

\section{Internet business environment architecture based on Web}

WoTSE model is different from the conventional OSI model, is not strictly a function of the details of the lower shielding concrete. In WoTSE model, generate intelligent network devices based networking application development and usability barriers and horizontal stacking and gradually increased [5]. Ability WoTSE structural levels, including equipment to access and abstraction layer, resource aggregation and intelligent services on the basis of three levels of support layer. Network Business Environment Web-based hierarchical model shown in Figure 2.

Device access and the ability to abstract layer is the first layer WoTSE structure, from the application point of view, its main role is to ensure that the application can continue to access different physical devices to provide relevant data and service resources. Seamlessly integrated into the network equipment proposed scheme of things and use the rest style of architecture and design reference Web standards. This layer provides the basic service capabilities without the Internet of Things devices connected to the Internet can also be a function of networking equipment, services, provide external open service based on other abstract principles. Typically it uses things in the 
intelligent gateway devices or integrated directly access the Internet. In addition, this layer also should be combined with the characteristics of the Internet of Things applications provide advanced data management features such as caching and scheduling. Web developers to access the device abstraction layer, and the ability to invoke a Web API node can directly access the network device and the service-related data, lower the threshold. In the case of a more complex business processes, however, only rely on services provided by the first layer of application development is still very high threshold, which requires resource aggregation layer to provide certain services. The main purpose of resource aggregation layer using business process engine and polymerization to produce resource-oriented business services. By arrangement, reusable business processes to achieve polymer networking equipment resources and business between applications and crashes, interdisciplinary cooperation.

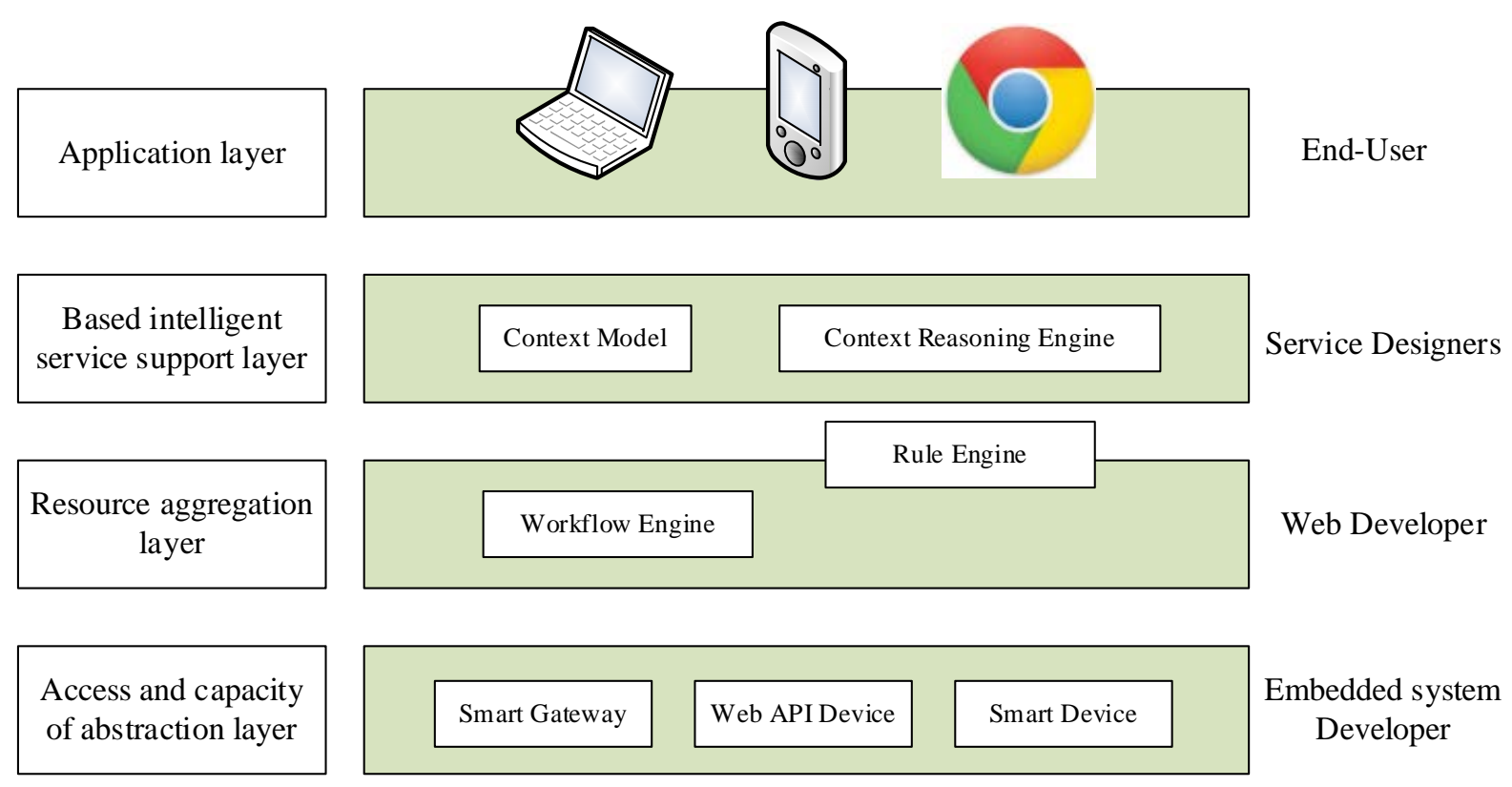

Figure 2. Internet business environment architecture based on Web

\section{The Internet of things application architecture design based on Web}

In this section, we mainly WoTSE concrete structures from the perspective of a particular functional entity. According WoTSE three-tier structure, it can be divided into functional entities know the service gateway, registration, resource discovery service composition and application generation, and context modeling and execution. WoTSE functional entities architecture shown in Figure 3.

Things device can automatically environmental consciousness to obtain information about the physical world, information processing and analysis can provide personalized and intelligent services for users. WoTSE first and second floor of the structure is provided with a lower threshold for developers to access and aggregation service capacity of these resources for networking device information, but the implementation of intelligent services still require the user based on their specific application requirements forms processing, selection of basic data and services. This process takes time to build a more professional tool that enables developers threshold remains high. So basic intelligence service support layer is the main function of the environment to provide developers based resource-oriented modeling and reasoning services to automate and intelligent services. 


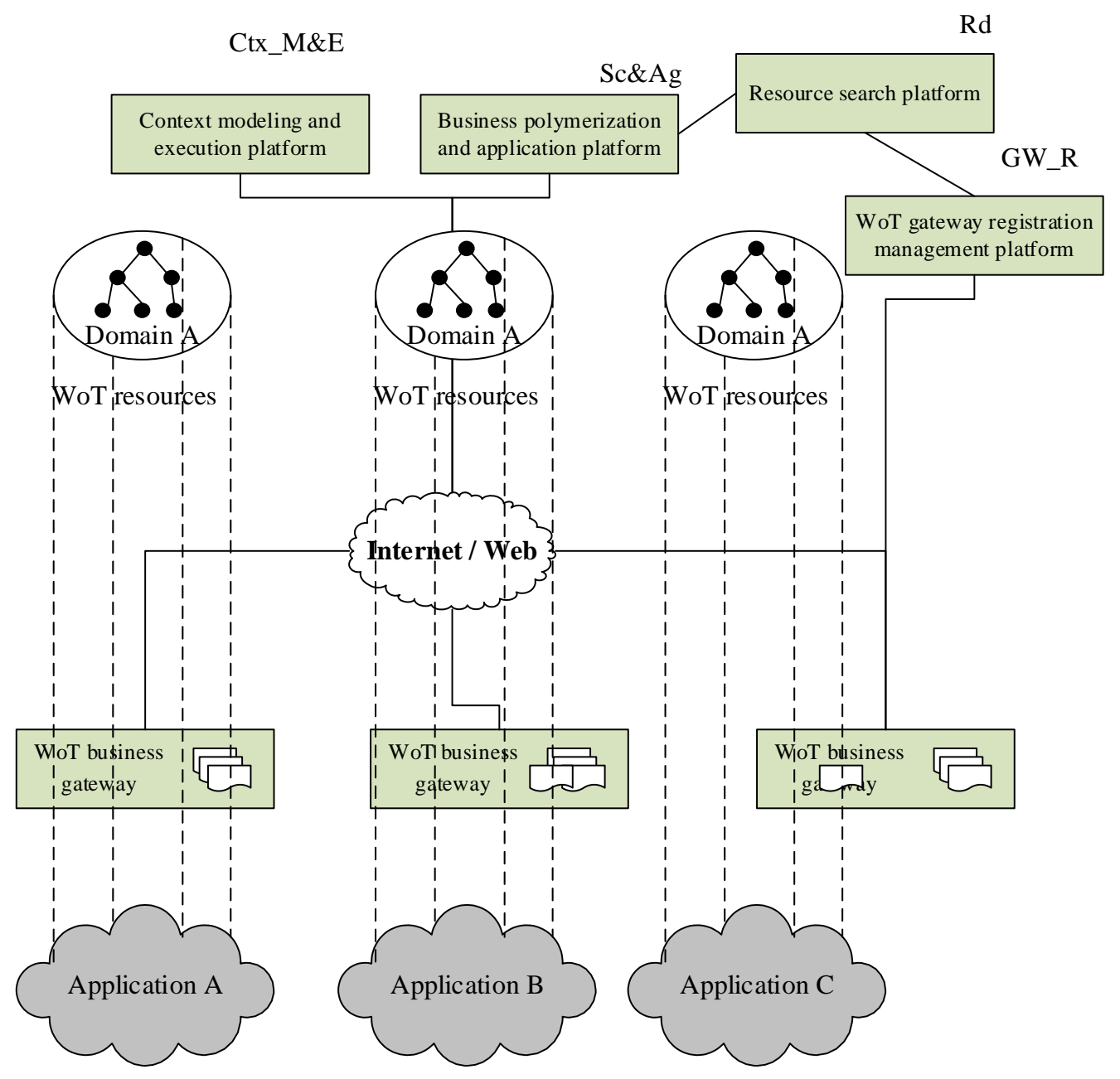

Figure 3. Internet business environment architecture based on Web

\section{Conclusion}

Pan application of Internet technology for the information society, greatly improving efficiency, some impact on people's lives. However, due to the existence of things devices and platforms, strong heterogeneity, high coupling and poor problem, so that debris networking applications, the status quo long development cycles and high threshold. According to the above problem, this Web-based business environment, architecture and key technologies studied, WoTSE architecture model design. WoTSE architecture model to solve the problem long development cycles and things high threshold is very effective.

\section{References}

[1] D. Miorandi, S. Sicari, F. De Pellegrini: Ad Hoc Networks, Vol.10 (2012) No.7, p. 1497.

[2] L.D. Xu: International Journal of Production Research, Vol. 49 (2011) No.1, p.183.

[3] D. Guinard, V. Trifa, S. Karnouskos: Services Computing, Vol.3 (2010) No.3, p. 223.

[4] L. Atzori, A. Iera, and G. Morabito: Computer networks, Vol.54(2010) No.15, p. 2787.

[5] S.E. Fawcett, G.M. Magnan, and M.W. McCarter: Supply Chain Management: An International Journal, Vol. 13 (2008) No.1, p.35. 